\title{
Twenty-Five Years of End-User Searching, Part 2: Future Research Directions
}

\author{
Karen Markey \\ School of Information, 1085 South University Avenue, University of Michigan, \\ Ann Arbor, MI 48109-1107. E-mail: ylime@umich.edu
}

\begin{abstract}
This is the second part of a two-part article that examines 25 years of published research findings on end-user searching of online information retrieval (IR) systems. In Part 1 (Markey, 2007), it was learned that people enter a few short search statements into online IR systems. Their searches do not resemble the systematic approach of expert searchers who use the full range of IR-system functionality. Part 2 picks up the discussion of research findings about end-user searching in the context of current information retrieval models. These models demonstrate that information retrieval is a complex event, involving changes in cognition, feelings, and/or events during the information seeking process. The author challenges IR researchers to design new studies of end-user searching, collecting data not only on system-feature use, but on multiple search sessions and controlling for variables such as domain knowledge expertise and expert system knowledge. Because future IR systems designers are likely to improve the functionality of online IR systems in response to answers to the new research questions posed here, the author concludes with advice to these designers about retaining the simplicity of online IR system interfaces.
\end{abstract}

\section{Introduction}

The first part of this two-part article reviews 25 years of published studies on end-user searching of online IR systems (Markey, 2007). It demonstrates that examining enduser searching by the search features people use everyday paints a simplistic picture of end-user searching that resembles the original classic model of online information retrieval in which one-time interactions between user and system figure prominently (Shannon \& Weaver, 1948). Since the mid-1960s, researchers have recognized that information retrieval is an iterative process between user and system. "[It] is a developing, unfolding sort of thing; it is

Received August 30, 2006; accepted August 30, 2006

C 2007 Wiley Periodicals, Inc. • Published online 16 April 2007 in Wiley InterScience (www.interscience.wiley.com). DOI: 10.1002/asi.20601 really an educational process-the more one knows, the more varied is the information that becomes acceptable and useful" (Vickery, 1965, p. 10).

Part 2 begins with an overview of three current theoretical models of the IR process. These models deserve explanation here because they recognize that information retrieval is not a one-stop event-people search repeatedly for the same topic of interest and their searches involve changes in cognition, feelings, and/or events of the information seeking process. The author poses new research questions that will go the next mile toward increasing our understanding about end-user searching of online IR systems. She concludes with advice to future IR system developers about retaining the simplicity of online IR system interfaces while adding improvements to these systems that will help end users find the information that satisfies their information needs.

\section{Current Information Retrieval Models}

Bates' (1989a) berrypicking model introduces bit-at-a-time retrieval. Retrieval is not a direct route from information need to final retrieved set. Instead, the search changes direction, pauses, and meanders as the user reads retrieved documents, follows up on leads, and responds to shifts in thinking. New information gives information seekers new ideas, new directions to pursue, and a new conception of their information needs. Kuhlthau's (1993) information search process (ISP) model divides information seeking into seven stages-initiation, selection, exploration, formulation, collection, presentation, and assessment - that are named for the primary task to be accomplished at each point in the process. Information seekers engage the stages recursively, moving back and forth between them, depending on the situation. When users are engaged in an extensive inquiry project, their thoughts evolve from vague and unclear to focused and personalized; their actions change from general and exploratory to specific and comprehensive; and their feelings emerge from uncertain and hesitant to interested and directed. 
The multiple information seeking episodes (MISE) model explains why people search repeatedly for the same information need across multiple episodes (Lin \& Belkin, 2000, p. 140): (a) the problem transmutes: the original problem is enriched, polished, expanded with new concepts, and changes its texture from the original to the transmuted state; (b) the problem spawns subproblems: the original problem spawns subproblems that have a higher priority than the original problem; (c) the problem transits problem: the original problem changes from one problem A to another B; (d) the problem rolls back: the original problem that the user had resolved turns out not to be resolved; (e) the answer to the problem is lost: the user loses the solution to the original problem; (f) the problem remains unanswered: the user cannot find a satisfactory solution to the problem, $(\mathrm{g})$ the problem is cultivated: the user continues to stay abreast of new developments pertaining to the original problem, and (h) the problem is anticipated: the user restarts the information seeking process due to external or internal pressures.

\section{Research Questions for Future Studies of End-User Searching}

Accompanying model development has been a host of empirical studies in which researchers explicitly demonstrate that people search repeatedly to satisfy their information needs. Robertson and Hancock-Beaulieu (1992) single out a set of end users who searched the Okapi online catalog repeatedly for the same topic over an extended period. Huang (1992) reports that 19 of 44 end users conducted multiple searches of online bibliographic databases for the same topic during the course of an academic semester. Spink (1996) interviews 200 end users who have just completed searches of online catalogs and CD-ROM databases and learns that just over half (56.5\%) have conducted multiple searches for their topics of interest. Asking users who conduct multiple searches to characterize their progress in ISP stages, Spink (1996, p. 607) reports that 55\% are in the initiation stage and a little under half are in these later stages, selection (5\%), exploration (18\%), formulation (5\%), or collection (17\%). Seventy percent of the users who conducted multiple searches tell Spink that they modified their search terms and strategy in their subsequent searches. Rieh (2003) discovers that end users break up their leisure time at home by searching the Web for 10 to 15 minutes at a time; they do multiple searches for certain topics of interest, "especially when the task [i]s one important to them or one on which they [will] to spend a lot of money (e.g., buying a house, finding a good place for vacation, moving to another area, and buying a digital camera" (p. 259). Additional studies explore the multiple searches phenomenon, but their researchers use intermediary searchers to search online IR systems (Spink, Griesdorf \& Bateman, 1999; Spink, Wilson, Ford, Foster, \& Ellis, 2002) or deliberately instruct end users to conduct multiple searches as part of a search task (Vakkari, 2001).

\section{Studies of Multiple Search Sessions}

Studies of system-feature use could benefit from studies of multiple search sessions and visa versa. The closest combination of the two is a 2-year study of humanities scholars who were trained in the use of the Dialog command-based retrieval system prior to being given unlimited access to Dialog for conducting their own searches (Siegfried, Bates, \& Wilde, 1993). Although the researchers log scholars' Dialog searches, they do not distinguish between the system features that scholars used on their initial or subsequent searches for the same topic of interest.

Combine the two study types and future researchers would be able to answer a host of new research questions:

1. Are the system features that end users enlist in their initial searches for a topic of interest the same as the system features they enlist in subsequent searches for the same topic?

2. To what extent do end-users' subsequent searches duplicate previous actions, especially with regard to their search terms?

3. Would users like to review their previous searches for a topic, resume them from the point at which they terminated them, or would they prefer systems to summarize previous search(es)? What summary information would be most useful, for example, databases(s) searched, search term(s) used, retrievals seen? Would the availability of summary information reduce user duplication of previous actions?

4. Are users more likely to use advanced system features when they perform subsequent searches for the same topic?

5. Characterize subsequent searches using the renewal reasons in the MISE model (Lin \& Belkin, 2000, p. 140). Then determine whether the system features that end users enlist in these subsequent searches are the same across all eight reasons.

6. If users make efforts to find additional material on their topics, for example, invoking renewal reasons such as roll back, anticipated, or unanswered problems on subsequent searches, would they welcome direct system intervention that puts the burden on the system to accomplish these tasks automatically or semiautomatically with their assistance?

7. If so few users perform Boolean searches and perform them correctly on both their initial and subsequent searches, should systems replace Boolean searching with alternative approaches and reserve Boolean searching for the systems that intermediary searchers use exclusively?

8. During the course of the end-user's searches for his or her topic of interest, what changes are apparent for relevance assessments generally? For relevance assessments based on the end-user's renewal reason? For relevance assessments of the same items retrieved in both the initial and subsequent searches? For relevance assessments based on the user's ISP stage?

9. Characterizing initial and subsequent searches by the user's ISP stage, are the system features that people use in their searches the same across all stages? 
10. Do users try to learn more about using online IR systems? What renewal reason or what ISP stage is in play during these occasions?

11. If certain ISP stages are connected to a user's attempts to gather additional search terms, find relevant retrievals, or learn about system use, would these users welcome direct system intervention that puts the burden on the system to accomplish these tasks automatically or semiautomatically with user assistance?

12. Do users think that a trained intermediary searcher could do a better job finding relevant material on both their initial and subsequent searches for a topic? Are different degrees of satisfaction connected with each of the eight renewal reasons for subsequent searches?

Although answers to these research questions will be of interest to IR researchers, their value to system designers should be paramount. If there are stark differences between end-users' initial and subsequent searches, system designers should take notice because it may be an opportunity for them to make their systems more adaptive to user needs.

Consider the perceived self-service nature of today's online research tools. How simple it is to type a query into an IR system, wait a split second for a response, and click on links for the most promising retrievals. It is akin to placing one's fast-food order at the take-out window, driving around the building, and gathering up the order at the pick-up window. Because of such simplicity, users may not necessarily feel compelled to use much more than the basic features of these systems that produce retrievals. However, when users find themselves in certain situations, for example, failing to find relevant retrievals on subsequent searches, they may be more inclined to use or learn how to use system features that require more than a cursory glance or click of the mouse such as relevance feedback, related terms lists, saved search summaries, and current alert profiles.

Among IR researchers, Bates' (1989b) idea of a front-end system mind to assist users with search term selection is legendary and so is the extensive experimentation with automatic and system-assisted feedback by a Rutgers research team (Belkin et al., 2001). Although both features have been implemented in operational systems, end users have largely ignored them. Let us put research findings about systemfeature use and multiple search sessions to work by building systems that are sensitive to the progress users are making on their ongoing searches, intervene with complex search features that are likely to solve user problems, and monitor users to determine whether these complex features help them achieve their goals.

\section{More Indicators That Users Could Benefit From Direct System Intervention}

When researchers analyze end-users' failed searches, the number one problem is their initial choice of search terms (Debowski, 2001, p. 377; Hsieh-Yee, 1993, p. 169; Lucas \& Topi, 2002, p. 105; Sewell \& Teitelbaum, 1986, p. 241; Wildemuth \& Moore, 1995, p. 299). Instead of using a database's controlled vocabulary, users search for the first terms that come to mind. Failing to use the controlled vocabulary has an adverse effect on the precision of their searches and makes it impossible for users to enlist the vocabulary's special search features such as exploding terms, listing subheadings, and displaying term relationships. Researchers who compare end-user search performance to expert searchers remark on the various ways in which the latter enlist controlled vocabulary in their searches (Lancaster, Elzy, Zeter, Metzler, \& Low, 1994; Rudner, 2000; Sutcliffe, Ennis, \& Watkinson, 2000). A longtime reference librarian assisting users at the Library of Congress, Mann (2003) observes "Even minimal experience working with readers at a reference desk — or, better, standing over their shoulders at a computer terminal-will demonstrate very clearly that most [us]ers, lacking either prior instruction or point-of-use assistance, are simply incapable of coming up with the best terms" (p. 53).

The initial query that end users enter into online IR systems is usually a broad-based concept or idea, and they then follow up with words and phrases that are more specific (Markey \& Demeyer, 1986, p. 158; Rieh \& Xie, 2001, p. 251; Vakkari, Pennaman, \& Serola, 2003, p. 459). If users persevere, "flip-flopping" ensues, that is, following up an initial broad-based concept with a specific term, flipping back to a broad-based concept, flopping to a specific term, adding a new term to express a narrower concept or an entirely new concept, and so on. Researchers have identified a variety of flip-flopping patterns. Except for the general-to-specific pattern, no one pattern is especially prevalent (Rieh \& Xie, 2001; Vakkari et al., 2003; Wildemuth, 2004; Wildemuth \& Moore, 1995). "Incredulous repetitions," repeatedly entering the same query, is another phenomenon that researchers have observed throughout the 25 years of end-user searching (Debowski, 2001; Jones, Cunningham, McNab, \& Boddie, 2000; Mischo \& Lee, 1987; Peters, 1993; Silverstein, Marais, Henziger, \& Monicz, 1999; Spink et al., 2004; Trzebiatowski, 1984). Peters (1993) speculates that "users engaging in this type of behavior $\mathrm{d}[\mathrm{o}]$ not believe or accept the results of the first search statement, particularly since the phenomenon frequently occur[s] when null sets [are] being retrieved" (p. 49).

Valentine (2001) describes end-user behavior that neither is targeted nor direct: "Students often use very chaotic, what they themselves term 'random,' methods for finding materials for their papers. A characteristic comment [i]s: 'I felt kind of aimless, kind of like shooting in the dark, you're going to get something eventually" (p. 112). With regard to the search terms users enter, Debowski (2001) observes the high and frenetic levels of effort on the part of online searchers. "It [is] evident that they spen[d] more time inputting, rather than planning a suitable search process. There [is] little evidence of search quality assessment ... with most entering the next search statement very rapidly. Despite the rapidity of the search entries, many enter few terms, preferring to reenter the same terms repeatedly... [Users] who search without a solid foundation fail to gain a stronger understanding of the search process. Instead, they appear to develop 
further erroneous habits as they continue" (p. 378). Other researchers observe the same haphazard behavior (Fidel et al., 1999; Halcoussis, Halverson, Lowenberg, \& Lowenberg, 2002; Hill, 1997; Rieh, 2003; Wallace, Kupperman, Krajcik, \& Soloway, et al., 2000).

Land and Greene (2000) attribute these types of user searching difficulties to low levels of metacognitive knowledge, "the process of reflecting on or monitoring the effectiveness of the search process and then refining the process when necessary" (p. 57). Metacognitive knowledge includes sequencing knowledge, which, if present at higher levels, enables searchers to order classes of information artifacts in an overall search plan (Bhavnani, 2001). Debowski (2000) adds " $[\mathrm{H}]$ igh levels of repetitive recycling, errors and frenetic search inputs all reduce [people's] capacity to develop effective search qualities. Instead, their activities reflect a focus on ad hoc activity, rather than constructive, premeditated search strategy" (p. 378). Patternless meandering, increasing numbers of errors, flip-flopping on the formulation of successive queries, incredulous repetitions, and brief stays within states are indicative of searchers with low metacognitive knowledge.

These situations are the occasion for more opportunities for systems to monitor user activity so they can intervene with assistance, in this case, with vocabulary assistance. Systems can be programmed to determine whether users are flip-flopping, entering a succession of errors, submitting incredulous repetitions, or displaying high levels of frenetic activity. Before embarking on a system design campaign for direct system intervention, let us first try to pinpoint when users experience vocabulary difficulties so we know when they would be receptive to such assistance. Combining the two study types, future researchers could start with answers to these half dozen research questions:

1. When conducting their initial search for a topic of interest, are people's search terms broader, narrower, related, different, or exact representations of their topics of interest? What ISP stage are they experiencing?

2. When conducting subsequent searches for a topic of interest, are people's search terms broader, narrower, related, different, or exact representations of their topics of interest? What ISP stage are they experiencing and what renewal reason in the MISE Model best describes their subsequent search?

3. When are incredulous repetitions most likely to occur, taking into account what ISP stage users are experiencing, whether they are performing an initial or subsequent search, and, in the case of the latter, what renewal reason best describes their search?

4. When is flip-flopping most likely to occur, taking into account what ISP stage users are experiencing, whether users are performing an initial or subsequent search, and, in the case of the latter, what renewal reason best describes their search?

5. When do domain experts exhibit low levels of metacognitive knowledge, in terms of the ISP stage they are experiencing, whether they are performing an initial or subsequent search, and, in the case of the latter, the renewal reason that best describes their search?

6. If double-double experts (domain experts who are also expert search intermediaries) ever exhibit low levels of metacognitive knowledge, what is happening in terms of the ISP stage they are experiencing, their knowledge of the topic they are searching, whether they are performing an initial or subsequent search, and, in the case of the latter, the renewal reason that best describes their search?

Direct system intervention is not especially novel nor unrealistic. PaperChase (Horowitz \& Bleich, 1981), one of the first online IR systems that offered Medline searching to end users, automated the VARY tactic (Bates, 1979, p. 208) by suggesting Medical Subject Headings (MeSH) and Subheadings for the free-text terms users enter and substituting them in place of non-MeSH terms (King, 1991, p. 371). Ovid Medline, our present-day successor to PaperChase, does the same thing. Google is discreet about its suggested correction of spelling errors in user queries. Although the Web features no controlled vocabulary, search engines could "borrow" from databases that feature controlled vocabularies. Taking a cue from how Google scrutinizes user queries for spelling errors, Web search engines could monitor queries for matches of controlled vocabulary terms, and, when systems spot users in sticky situations (e.g., exhibiting frenetic behavior or entering incredulous repetitions), they could respond with assistance from these vocabularies.

Users cannot always describe what they really want or have in mind. Belkin (1980) commented on this over 25 years ago, "A problem is recognized, and it is recognized that information might be necessary to resolve the problem, but precisely because of the inquirer's lack of knowledge about the problem area, it is impossible [for users] to specify what would resolve it" (p. 137). Yet, users are able to recognize what they want or do not want during the course of the search. What information systems report back to users should make for easy reviewing and instantaneous recognition of relevant possibilities. Some systems have experimented with new and innovative reporting-back featuresthe defunct Northern Light search engine used search folders to characterize retrievals and the new Grokker search engine uses web visualization category maps (Groxis, 2005; Markoff, 2005, p. C3).

Expecting systems to diagnose the particular problem users are experiencing and give assistance tailored to an information seeker's special needs is a tall order. A good starting point may be the three levels of search activitiesmoves, tactics, and strategems - that Bates (1990) suggests for automation in an article she published long before the availability of today's online IR systems with their easyto-use graphical-user interfaces. Much experimentation is needed to determine when users want intervention, the types of intervention they will tolerate, and their preferred reporting formats. 


\section{Accounting for Domain Knowledge}

Because of the multitudinous ways of expressing topics (Furnas, Landauer, Gomez, \& Dumais, 1987), search strategies that require people to enter topical queries are especially prone to disaster. Domain knowledge experts who are searching in their area of expertise avoid search strategies that require them to enter topical queries (Bath University, 1972; Drabenstott, 2003; Ellis, 1989; Land \& Greene, 2000; Stenstrom \& McBride, 1979; Stoan, 1984; Van Styvendaele, 1977; Wood \& Bower, 1969;) and instead enlist strategies that that require objective information such as names of authors, projects, and article and journal titles. Ellis (1989) and Bates (1989a) describe these strategies: (a) searching for the names of authors, sponsoring organizations, projects, research centers, etc., who are active contributors to solving a problem in hand, (b) chasing the footnotes in a relevant item in hand, (c) searching for authors who have cited a relevant item in hand, and (d) the journal run, scanning issues of the same journal that published a relevant item in hand. Because domain experts avoid conducting subject searches in online IR systems, they never have to put into words exactly what interests them.

Ultimately, the goal of domain expert strategies is high precision because they severely limit the playing field. Relying on the precision of their strategies, experts then engage in recognition activities, looking for one or more clues-a title word or phrase, a sentence in an abstract, a name of an author, variable, test, research center-that are promising in terms of describing their interests because retrievals are few and almost on target right from the beginning. For example, enlisting the journal run strategy, domain experts put the spotlight on a single journal that because it has previously published relevant articles on the topic that interests them, it will publish additional ones.

The few times that domain novices enlist the search strategies of domain experts are due to serendipity, perseverance, trial-and-error, or a combination of all three (Drabenstott, 2003, p. 836). Novices who model the successful behaviors of domain experts may be able to make the leap from their inefficient subject search strategy to expert strategies. For example, Drabenstott (2003, p. 846) tells how one instructor gives his novice student assistant a list of sources to check, e.g., author names, journal titles, and organization names. If these sources pan out, the novice may be inclined to initiate this strategy on his own later. Unfortunately, most novices stumble upon domain-expert strategies. For example, a domain novice browses a list of electronic journal titles beginning with the letter A and selects the Archives of Sexual Behavior because it describes his topic, and prints a few relevant articles from recent issues. Terminating his search, the novice says he must remember this journal's name for future reference, but he does not mention that he would keep the strategy of browsing journal titles and issue contents in mind for the future (Drabenstott, 2003, p. 846).
When future researchers combine the two study types, their experimental designs should control for domain knowledge. Imagine answers to these research questions:

1. When searching in their areas of expertise, do domain experts ever conduct subject searches? If they do, are these searches their initial or subsequent searches of a topic, what ISP stage are they experiencing, and, in the case of subsequent searches, what renewal reason in the MISE model best describes these searches?

2. When searching outside their areas of expertise, do domain experts conduct primarily subject searches? If they do, are these searches their initial or subsequent searches of a topic, what ISP stage are they experiencing, and, in the case of subsequent searches, what renewal reason in the MISE model best describes these searches?

3. If domain experts who are searching outside their areas of expertise enlist the strategies one would expect from a domain expert in this particular domain (e.g., the journal run, chasing footnotes, author searching), are these their initial or subsequent searches of the topic? What ISP stage are they experiencing, and, in the case of subsequent searches, what renewal reason in the MISE model best describes these searches?

4. What advanced search features (e.g., relevance feedback, saved searches, current alert profiling) characterize domain expert searches in and outside their areas of expertise?

5. To what extent do nondomain experts ever enlist the strategies one would expect from a domain expert (e.g., the journal run, chasing footnotes, author searching), are these their initial or subsequent searches of the topic, what ISP stage are they experiencing, and, in the case of subsequent searches, what renewal reason in the MISE model best describes these searches?

6. If systems intervene with domain-expert strategies, would end users follow through? If these strategies helped end users achieve their search objectives, would end users actively seek these strategies out on their own at a later time?

When Bates and Ellis codified domain expert strategies in the late 1980s, it was not possible to build domain expert strategies into online systems because so few systems and databases were available to the public. Today's online IR environment is much more diverse and versatile and automating the four principal domain expert strategies-the journal run, author searching, cited-author searching, and footnote chasing is now possible. There are no easy answers when it comes to anticipating when people would be receptive to direct system intervention or determining which domain knowledge expert strategy systems should advise them to pursue.

Bhavnani et al. (2003) offers an alternative approach to direct intervention in all-purpose, encyclopedic Web search engines. His research team builds strategy hubs, custom interfaces that enlist domain expert search strategies for specialized topics. For example, a strategy hub that enlists the 
successful strategies of an expert Web shopper in search of the lowest prices on digital cameras would sequence three different types of Web sites to get the job done: (a) review sites to identify cameras and prices, (b) price comparison sites to compare prices, and (c) coupon sites to find discounts. Precursors to the Strategy Hub are the Book House for domain novices searching fiction (Pejtersen et al., 1995) and the Ohio State gateway for domain novices searching academic libraries (Tiefel, 1995).

Determining which domains would be the first candidates for strategy hub development may not be difficult because we could turn to the periodic surveys of Web users to learn typical tasks that take their time and attention (Fallows, 2004; Pew Research Center, 2003, 2005). Ultimately, building strategy hubs for the many typical information seeking tasks that occupy people would be a gargantuan undertaking because of the sheer number and variety of different tasks and the rate at which information sources and services are likely to change in these times of rapid technological change.

An alternative to the development of strategy hubs for the multitudinous variety of search tasks that occupies people's interests would be strategy hubs that encourage domain novices to generalize what they have learned regarding information seeking in one domain and apply it to a new and different domain for which no strategy hub exists. Then users could quickly and efficiently develop the information seeking strategies of domain experts on their own. This will not be easy. To date, we have not done a very good job instructing end users about online searching. Analyses of transaction logs demonstrate that end users do not consult online assistance nor has it transformed them into instant online searching experts who can restate their natural language queries in Boolean-based search expressions bearing correct Boolean operators, truncation, nested logic, proximity connectors, and syntax.

I have come to believe that making online IR systems more complicated with additional functionality, frequent and unanticipated interruptions in the form of direct system intervention, and detailed instructions and tutorials in system use, is not the right way to proceed. Instead, let us think deeply about how IR systems could covertly teach people about online searching at the same time they use the system to conduct their business. What can we learn from comparable pursuits such as gaming, online chat, text messaging, and music-to-go? What other computer-based activities capture people's attention for long periods and keep them coming back for more? Let us use what we learn from these observations to build future systems that covertly teach and advise at the same time their users conduct business.

\section{Closing Caveats}

Transaction logs have been a very useful tool for giving IR researchers and system developers a snapshot of end-user activity. Logs have several advantages that have made them especially desirable as a research tool. For the purposes of this article, logs are an unobtrusive approach to data collection, researchers can write custom programs to count, tally, and compare observations making it possible to analyze millions of cases in one fell swoop, and, for local systems such as online catalogs and locally mounted online databases, they are usually available for the asking. Unfortunately, they will not be sufficient for answering the research questions enumerated in this article. Information retrieval researchers would have to supplement their data-collection activities with other methods and most of those methods would be more obtrusive than transaction logs; however, to understand what people are searching, why they change their search terms, whether they are conducting an initial or subsequent search on a topic, what renewal reasons in the MISE model pertain to users' subsequent searches, and what ISP stages they are experiencing, IR researchers must have some form of contact with users. At academic institutions, they could use authorization data to single out faculty from undergraduate and graduate students to answer some of the research questions connected with domain expertise enumerated in this article; however, it may be difficult for IR researchers to determine when faculty are searching in the areas of expertise and when they are searching outside of them so contact may still be necessary.

Over the years, many IR researchers have recruited intermediary searchers and/or graduate students at schools of information and library studies to serve as subjects in empirical studies. Although such people may be readily accessible and downright eager to participate, at the very least, they are cognizant of the Boolean search strategies and Boolean search functionality that everyday people rarely use. Intermediary searchers are and students are learning to be double-double experts, that is, experts in the study, teaching, and practice of online information retrieval. The few research projects that include double-double experts in their experimental design should be demonstrating just how different these experts search from ordinary people, instead of using these experts to generalize about end-user searching generally.

Finally, let us avoid research protocols that assign tasks to end users. As much as possible, researchers should design experiments that capture what end users really do, not what researchers want or expect them to do. Although collecting information on ISP stages and renewal reasons will require researchers to maintain contact with users, researchers need to be inventive about devising unobtrusive methods so that they collect reliable data on the information seeking habits of end users.

\section{Conclusion}

People enter a few short search statements into online IR systems. Generally, their queries bear two to four words. Boolean operators are uncommon. Boolean operators are even few in number in the searches of end users who receive training in system use. End users rarely use advanced system features and when they do, they are quite likely to use them incorrectly. Although research findings demonstrate that end 
users are not conducting sophisticated online searches, the vast majority are satisfied with their searches. In fact, percentages of users who express satisfaction with the results of their searches reach into the high seventies and beyond.

Characterizing end-user searches by the search features people use everyday paints a bleak picture of end-user searching. Because it resembles the original classic model of online information retrieval in which one-time interactions between user and system figure prominently, I discuss findings in the context of information retrieval models that paint a much more complex picture of end-user searching, involving changes in cognition, feelings, and/or events during the information seeking process. I challenge IR researchers to design new studies of end-user searching, collect data on both system-feature use and multiple search sessions, and control for variables such as domain knowledge expertise and expert system knowledge. Information retrieval researchers should be occupied for some time to come by the two dozen new research questions raised in this article. Finally, I invite IR system designers to put future research findings to work by building systems that are sensitive to the progress users are making in their ongoing searches, intervene with complex search features that are likely to solve user problems, and monitor users to determine whether these complex features help them achieve their goals.

\section{References}

Bates, M.J. (1979). Information search tactics. Journal of the American Society for Information Science, 30, 205-214.

Bates, M.J. (1989a). The design of browsing and berrypicking techniques for the online search interface. Online Review, 13, 407-424.

Bates, M.J. (1989b). Rethinking subject cataloging in the online environment. Library Resources and Technical Services, 33, 400-412.

Bates, M.J. (1990). Where should the person stop and the information search interface start? Information Processing \& Management, 26, $575-591$.

Bath University. (1972). Experimental information service in the social sciences 1969-1971: Final report. Bath, UK: Author.

Belkin, N.J. (1980). Anomalous states of knowledge as a basis for information retrieval. Canadian Journal of Information Science, 5, 133-143.

Belkin, N.J., Cool, C., Kelly, D., Lin, S.-J., Park, S.Y., Perez-Carbllo, J., et al. (2001). Exploration, design and evaluation of support for query reformulation in interactive information retrieval. Information Processing and Management, 37, 403-434.

Bhavnani, S.K. (2001). Important cognitive components of domain-specific search knowledge. In E.M. Voorhees \& L.P. Buckland (Eds.), The Tenth Text Retrieval Conference (pp. 571-578). Washington, DC: Department of Commerce and National Institute of Standards and Technology.

Bhavnani, S.K., Bichakjian, C.K., Johnson, T.M., Little, R.J., Peck, F.A., Schwartz, J.L., et al. (2003). Strategy hubs: Next-generation domain portals with search procedures. In CHI Conference Proceedings: Conference on Human Factors In Computing Systems (pp. 393-400). New York: Association for Computing Machinery.

Debowski, S. (2001). Wrong way: Go back! An exploration of novice search behaviours while conducting an information search. The Electronic Library, 19, 371-382.

Drabenstott, K.M. (2003). Do non-domain experts enlist the strategies of domain experts? Journal of the American Society for Information Science and Technology, 54, 836-854.

Ellis, D. (1989). A behavioural approach to information retrieval system design. Journal of Documentation, 45, 171-212.
Fallows, D. (2004). Search engine users: Internet searchers are confident, satisfied, and trusting — but they are also unaware and naïve. Washington, DC: Pew Internet \& American Life Project. Retrieved March 2, 2007, from http://www. pewinternet.org/PPF/r/146/report_display.asp

Fidel, R., Davies, R.C., Douglas, M.H., Holder, J.K., Hopkins, C.J., Kushner, E.J., et al. (1999). A visit to the information mall: Web searching behavior of high school students. Journal of the American Society for Information Science and Technology, 50, 24-37.

Furnas, G.W., Landauer, T.K., Gomez, L.M., \& Dumais, S.T. (1987). The vocabulary problem in human-system communication. Communications of the ACM, 30, 964-971.

Groxis, Inc. (2005). Grokker: A new way to look at search. Retrieved March 2, 2007, from http://www.grokker.com/

Halcoussis, D., Halverson, A.L., Lowenberg, A.D., \& Lowenberg, S. (2002). An empirical analysis of web catalog user experiences. Information Technology and Libraries, 21, 148-157.

Hill, J.R. (1997). The World Wide Web as a tool for information retrieval: An exploratory study of users' strategies in an open-ended system. School Library Media Quarterly, 25, 229-236.

Horowitz, G.L., \& Bleich, H.L. (1981). PaperChase: A computer program to search the medical literature. New England Journal of Medicine, 305, 924-930.

Hsieh-Yee, I. (1993). Effects of search experience and subject knowledge on the search tactics of novice and experienced searchers. Journal of the American Society for Information Science and Technology, 44, $161-174$.

Huang, M.-H. (1992). Behavior of end-users in online searching. Unpublished doctoral dissertation, University of Maryland, College Park, MD.

Jones, S., Cunningham, S.J., McNab, R., \& Boddie, S. (2000). A transaction $\log$ analysis of a digital library. International Journal of Digital Libraries, 3, 152-169.

King, N.S. (1991). Search characteristics and the effects of experience on end users of PaperChase. College \& Research Libraries, 52, 360-374.

Kuhlthau, C.C. (1993). Seeking meaning: A process approach to library and information services. Norwood, NJ: Ablex.

Lancaster, F.W., Elzy, C., Zeter, M.J., Metzler, L., \& Low, Y.-M. (1994). Searching databases on CD-ROM: Comparison of the results of end-user searching with results from two modes of searching by skilled intermediaries. RQ, 33, 370-386.

Land, S.M., \& Greene, B.A. (2000). Project-based learning with the World Wide Web: A qualitative study of resource integration. ETR\&D, 48, 45-67.

Lin, S.-J., \& Belkin, N.J. (2000). Modeling multiple information seeking episodes. In M.K. Roderer \& D.H. Kraft (Eds.), Proceedings of the 63rd Annual Meeting of the American Society for Information Science and Technology (Vol. 37, pp. 133-147). Silver Spring, MD: American Society for Information Science and Technology.

Lucas, W., \& Topi, H. (2002). From and function: The impact of query term and operator usage on Web search results. Journal of the American Society for Information Science and Technology, 53, 95-108.

Mann, T. (2003). Why LC subject headings are more important than ever. American Libraries, 34(9), 51-54.

Markey, K. (2007). Twenty-five years of end-user searching, part 1: Research findings. Journal of the American Society for Information Science and Technology, 58(8), 1071-1081.

Markey, K., \& Demeyer, A.N. (1986). Dewey Decimal Classification Online Project: Evaluation of a library schedule and index integrated into the subject searching capabilities of an online catalog. Dublin, $\mathrm{OH}$ : OCLC Online Computer Library Center.

Markoff, J. (2005, May 9). Your Internet search results, in the round. New York Times, p. C3.

Mischo, W.H., \& Lee, J. (1987). End-user searching of bibliographic databases. In M.E. Williams (Ed.), Annual review of information science and technology (pp. 227-263). Washington, DC: American Society for Information Science.

Pejtersen, A.M. (1992). The Book House: An icon based database system for fiction retrieval in public libraries. In B. Cronin (Ed.), The marketing of library and information services (Vol. 2, pp. 572-591). London: Aslib. 
Peters, T.A. (1993). The history and development of transaction log analysis. Library Hi Tech, 11(2), 41-66.

Pew Research Center. (2003). America's online pursuits: The changing picture of who's online and what they do. Washington, DC: Author. Retrieved September 5, 2005, from http://www.pewinternet.org/reports/toc.asp? Report $=106$

Pew Research Center. (2005). Internet: The mainstreaming of online life. In Trends 2005 (pp. 65-77). Washington, DC: Author. Retrieved September 5, 2005, from http://pewresearch.org/trends/

Rieh, S.Y. (2003). Investigating web searching behavior in home environments. In R.J. Todd (Ed.), Proceedings of the 66th Annual Meeting of the American Society for Information Science and Technology (Vol. 40, pp. 255-264). Silver Spring, MD: American Society for Information Science and Technology.

Rieh, S.Y., \& Xie, H.I. (2001). Patterns and sequences of multiple query reformulations in web searching: A preliminary study. In E. Aversa \& C. Manley (Eds.), Proceedings of the 64th Annual Meeting of the American Society for Information Science and Technology (Vol. 38, pp. 246-255). Silver Spring, MD: American Society for Information Science and Technology.

Robertson, S.E., \& Hancock-Beaulieu, M.M. (1992). On the evaluation of IR systems. Information Processing \& Management, 28, 457-466.

Rudner, L. (2000). Who is going to mine digital library resources? And how? D-Lib Magazine 6, 5. Retrieved, from http://www.dlib.org/dlib/may00/ rudner/05rudner.html

Sewell, W., \& Teitelbaum, S. (1986). Observations of end-user online searching behavior over eleven years. Journal of the American Society for Information Science, 37, 234-245.

Shannon, C.E., \& Weaver, W. (1949). The mathematical theory of communication. Urbana, IL: University of Illinois Press.

Siegfried, S., Bates, M.J., \& Wilde, D.N. (1993). A profile of end-user searching behavior by humanities scholars: The Getty Online Searching Project Report No. 2. Journal of the American Society for Information Science, 44, 273-291.

Silverstein, C., Marais, H., Henziger, M., \& Monicz, M. (1999). Analysis of a very large web search engine query log. SIGIR Forum, 33(1), 6-12.

Spink, A. (1996). Multiple search sessions model of end-user behavior: An exploratory study. Journal of the American Society for Information Science, 47, 603-609.

Spink, A., Griesdorf, H., \& Bateman, J. (1999). A study of mediated successive searching during information seeking. Journal of Information Science, 25, 477-487.
Spink, A., Ozmutlu, H.C., \& Lorence, D.P. (2004). Web searching for sexual information: An exploratory study. Information Processing and Management, 40, 113-123.

Spink, A., Wilson, T.D., Ford, N., Foster, A., \& Ellis, D. (2002). Information seeking and mediated searching study. Part 3. Successive searching. Journal of the American Society for Information Science, $53,716-727$.

Stenstrom, P., \& McBride, R.B. (1979). Serial use by social science faculty: A survey. College \& Research Libraries, 40, 426-431.

Stoan, S.K. (1984). Research and library skills: An analysis and interpretation. College \& Research Libraries, 45, 99-109.

Sutcliffe, A.G., Ennis, M., \& Watkinson, S.J. (2000). Empirical studies of end-user information searching. Journal of the American Society for Information Science, 51, 1211-1231.

Tiefel, V. (1995). Library user education: Examining its past, projecting its future. Library Trends, 44, 318-338.

Trzebiatowski, E. (1984). End user study on BRS/Afterdark. RQ, 23, 446-450.

Vakkari, P. (2001). A theory of the task-based information retrieval process: A summary and generalisation of a longitudinal study. Journal of Documentation, 57, 44-60.

Vakkari, P., Pennanen, M., \& Serola, S. (2003). Changes of search terms and tactics while writing a research proposal: A longitudinal case study. Information Processing and Management, 39, 445-463.

Valentine, B. (2001). The legitimate effort in research papers: Student commitment versus faculty expectations. Journal of Academic Librarianship, $27,107-115$

Van Styvendaele, J.H. (1977). University scientists as seekers of information: Sources of reference to periodical literature. Journal of Librarianship, 9, 270-277.

Vickery, B.C. (1965). On retrieval system theory. London: Butterworths. Wallace, R., Kupperman, J., Krajcik, J., \& Soloway, E. (2000). Science on the web: Students online in a sixth-grade classroom. Journal of the Learning Sciences, 9, 75-104.

Wildemuth, B. (2004). The effects of domain knowledge on search tactic formulation. Journal of the American Society for Information Science and Technology, 55, 246-258.

Wildemuth, B., \& Moore, M.E. (1995). End-user search behaviors and their relationship to search effectiveness. Bulletin of the Medical Library Association, 83, 294-304.

Wood, D.N., \& Bower, C.A. (1969). The use of social science periodical literature. Journal of Documentation, 9, 108-122. 